\title{
SEMILATTICES WHICH ARE EMBEDDABLE IN A PRODUCT OF MIN INTERVALS
}

\section{J. W. STEPP}

AbSTRACr. In this paper we give necessary and sufficient conditions for a locally compact semilattice to be embeddable in a product of min intervals.

I. Introduction. In [2] J. D. Lawson gave conditions which guarantee that a topological semilattice have sufficiently many continuous homomorphisms into the min interval to separate points. However, a semilattice may have this property and not be embeddable in a product of min intervals (Example 1 and Example 2). The purpose then of this paper is to give conditions which will guarantee that a locally compact semilattice is embeddable in a product of min intervals.

II. Definitions and notations. Let $S$ denote a topological semilattice; that is, $S$ is a commutative idempotent topological semigroup with $x \leqq y$ if and only if $x y=x$. For each $x$ in $S$ let $M_{S}(x)$ $=\{y \in S \mid x \leqq y\}$ and let $L_{S}(x)=\{y \in S \mid y \leqq x\}$. For each nonempty subset $A$ of $X$ let $M_{S}(A)=\bigcup\left\{M_{S}(x) \mid x \in A\right\}$ and let $L_{S}(A)=$ $\mathrm{U}\left\{L_{S}(x) \mid x \in A\right\}$. Whenever there is no danger of confusion we write $M(-)$ instead of $M_{S}(-)$ and $L(-)$ instead of $L_{S}(-)$. A nonempty set $B$ of $S$ is called an increasing (decreasing) set if $M(B)=B(L(B)=B$ ). If $B$ is both an increasing set and a subsemilattice, then $B$ is called an increasing subsemilattice. A set $B$ is called convex if for each $x$ and $y$ in $B$ it follows that $M(x) \cap L(y) \subseteq B$. If $A \subseteq S$, then $\bar{A}$ denotes the topological closure of $A$ and $A^{\circ}$ denotes the interior of $A$. Let $M$ denote the min interval; that is, $M$ is the unit interval with multiplication given by $x y=$ g.l.b. $\{x, y\}$. Let $\operatorname{Hom}(S, M)$ denote the set of continuous homomorphisms from $S$ into $M$, and let $X_{S} M$ denote the product semilattice $X\left\{M_{f} \mid f \in \operatorname{Hom}(S, M)\right.$ and $\left.M_{f}=M\right\}$. For each $g$ in $\operatorname{Hom}(S, M)$ let $\mathrm{pr}_{\theta}$ denote the map from $X_{S} M$ onto $M_{g}$ defined by $\operatorname{pr}_{o}\left(\left(m_{f}\right)\right)=m_{g}$ for all $\left(m_{f}\right)$ in $X_{S} M$, and let $\Psi: S \rightarrow X_{S} M$ be the map defined by $\operatorname{pr}_{g}(\Psi(s))=g(s)$ for all $s$ in $S$ and all $g$ in $\operatorname{Hom}(S, M)$. Then $\Psi$ is called the evaluation map. Observe that $\Psi$ is injective if and only if $\operatorname{Hom}(S, M)$ separates points.

Received by the editors January 14, 1970.

AMS 1970 subject classifications. Primary 06A20; Secondary 20M10.

Key words and phrases. Locally compact semilattices, semilattices with small semilattices, min interval, locally convex semilattice. 
Definition 2.1. A semilattice is locally convex if the collection of open convex subsets form a base for the topology.

DEFINITION 2.2. A semilattice $S$ is $L U$-completely regular at $s$ if for each open neighborhood $U$ of $s$ there is a point $s_{0}$ in $U$ with $s \in M\left(s_{0}\right)^{\circ}$ such that if $B$ is a closed (in $S$ ) increasing subsemilattice contained in $M\left(s_{0}\right) \backslash\{s\}$, then there is an $f$ in $\operatorname{Hom}(S, M)$ such that $f(s)=0$ and $f(B)=1$. A semilattice is $L U$-completely regular if it is $L U$-completely regular at each of its points.

Definition 2.3. A semilattice is $L U$-finite at $s$ if for each open neighborhood $U$ of $s$ there is a point $s_{0}$ in $U$ with $s \in M\left(s_{0}\right)^{\circ}$ such that if $B$ is a closed (in $S$ ) increasing set contained in $M\left(s_{0}\right) \backslash\{s\}$, then there are closed increasing subsemilattices $B_{1}, \cdots, B_{n}$ satisfying $s \notin \bigcup_{i=1}^{n} B_{i}$ and $B \subseteq \bigcup_{i-1}^{n} B_{i}$. A semilattice is $L U$-finite if it is $L U$-finite at each of its points.

III. In this section we prove that a locally compact topological semilattice $S$ is embeddable in a product of min intervals if and only if $S$ is locally convex, $L U$-completely regular, and $L U$-finite. Throughout this section $S$ will denote a topological semilattice.

We now state some known results (Theorem 3.1 and Theorem 3.2) which we will use throughout this section. The proofs of these two theorems are due to J. D. Lawson [2].

TheOREM 3.1. Let $S$ have the property that if $x \in V$, an open, increasing subset of $S$, then there exists $y \in V$ such that $x \in M(y)^{\circ}$. If $A$ is a closed, decreasing subset and $b \in S \backslash A$, then there exists an $f$ in $\operatorname{Hom}(S, M)$ such that $f(A)=0$ and $f(b)=1$.

ThEOREM 3.2. Let $S$ be a compact topological semilattice. Then the following are equivalent:

(a) $\operatorname{Hom}(S, M)$ separates points.

(b) S satisfies the hypothesis of Theorem 3.1.

(c) For each $s$ in $S$ and each neighborhood $U$ of $s$ there is a neighborhood $V$ of $s$ which is a subsemilattice of $S$ such that $V \subseteq U$.

(d) The evaluation map $\Psi$ is an embedding.

Lемма 3.3. If $S$ is compact and $\operatorname{Hom}(S, M)$ separates points, then $S$ is LU-completely regular.

Proof. Let $s \in S$ and let $U$ be an open neighborhood of $s$. Since $S$ is compact, $S$ is locally convex [3, pp. 27, 48]. Thus there is an open convex neighborhood $V$ of $s$ with $V \subseteq U$. Then $M(V)$ is an open increasing set with $s \in M(V)$. Thus there is a point $s_{0}$ in $M(V)$ with $s \in M\left(s_{0}\right)^{\circ}$ (Theorem 3.2b). Since $s_{0} \in M(V)$, there is a point $t \in V$ with $t \leqq s_{0}$; since $s \in M\left(s_{0}\right)^{\circ}, s_{0} \leqq s$. Thus $t \leqq s_{0} \leqq s$ and since $V$ is convex 
it follows that $s_{0} \in V$. Since $V \subseteq U$, it follows that $s_{0} \in U$. Let $B$ be a closed (in $S$ ) increasing subsemilattice which is contained in $M\left(s_{0}\right) \backslash\{s\}$. Since $S$ is compact, $B$ is compact, and therefore $B$ has a zero. Let $b$ denote the zero for $B$. Then $b \notin L(s)$. Thus by Theorem 3.1 and Theorem 3.2b, there is an $f$ in $\operatorname{Hom}(S, M)$ with $f(b)=1$ and $f(L(s))=0$. It follows that $f(B)=1$ and $f(s)=0$.

LeMma 3.4. If $S$ is compact and $\operatorname{Hom}(S, M)$ separates points, then $S$ is LU-finite.

Proof. Let $s \in S$ and let $U$ be an open neighborhood of $s$. As in the proof of Lemma 3.3, there is a point $s_{0}$ in $U$ with $s \in M\left(s_{0}\right)^{\circ}$. Let $B$ be a closed (in $S$ ) increasing set which is contained in $M\left(s_{0}\right) \backslash\{s\}$. Then $B \cap L(s)=\varnothing$. For each $b$ in $B$ let $U_{b}$ be an open neighborhood of $b$ with $\bar{U}_{b} \cap L(s)=\varnothing$. By Theorem 3.2c, for each $b$ in $B$ there is a subsemilattice $V_{b}$ which is a neighborhood of $b$ with $V_{b} \subseteq U_{b}$. Then $V_{b} \cap L(s)=\varnothing$. Since $\bar{V}_{b}$ is compact, $M\left(\bar{V}_{b}\right)$ is a closed increasing subsemilattice with $M\left(\bar{V}_{b}\right) \cap L(s)=\varnothing$. The set $\left\{V_{b}^{\circ} \mid b \in B\right\}$ is a cover of $B$ by open sets and thus there is a finite subset $\left\{V_{1}, \cdots, V_{n}\right\}$ of $\left\{V_{b} \mid b \in B\right\}$ such that $B \subseteq \bigcup_{i=1}^{n} V_{i}$. Let $B_{i}=M\left(V_{i}\right)$. Then $B \subseteq \bigcup_{i=1}^{n} B_{i}$ and $s \notin U_{i=1}^{n} B_{i}$.

Lemma 3.5. Let $T$ be a compact topological semilattice, and let $S$ be an open subsemilattice of $T$.

(a) If $T$ is $L U$-completely regular, then $S$ is $L U$-completely regular.

(b) If $T$ is $L U$-finite, then $S$ is $L U$-finite.

Proof. (a) Let $s \in S$, and let $U$ be an open (in $S$ ) neighborhood of $s$. Since $T$ is compact, there is an open convex subset $V$ of $T$ which contains $s$ such that $V \subseteq S$. Again as in Lemma 3.3 there is a point $s_{0}$ in $V$ with $s \in M_{S}\left(s_{0}\right)^{\circ}$. Let $B$ be a closed (in $S$ ) increasing subsemilattice with $B \subseteq M_{S}\left(s_{0}\right) \backslash\{s\}$. Let $\bar{B}$ denote the closure of $B$ in $T$. Since $V$ is convex (relative to $T$ ), $M_{T}(\bar{B}) \cap L_{T}(s)=\varnothing$. By Theorem 3.1 and since $T$ is $L U$-completely regular, $\operatorname{Hom}(T, M)$ separates points; thus there is an $f$ in $\operatorname{Hom}(T, M)$ such that $f(s)=0$ and $f(b)=1$, where $b$ is the zero of the compact semilattice $M_{T}(\bar{B})$. Let $g=f \mid S$. Then $g(s)=0$ and $g(B)=1$, and $g \in \operatorname{Hom}(S, M)$. Thus $S$ is $L U$-completely regular.

(b) Let $s, U$, and $V$ be as in the proof of part (a). By Theorem 3.1 and since $T$ is $L U$-finite, $\operatorname{Hom}(T, M)$ separates points; thus by Lemma 3.4 there is a point $s_{0}$ in $V$ with $s \in M_{T}\left(s_{0}\right)^{\circ}$ such that if $A$ is a closed (in $T$ ) increasing set contained in $M_{T}\left(s_{0}\right) \backslash\{s\}$, then there are closed increasing subsemilattices $A_{1}, \cdots, A_{n}$ of $T$ with $s \notin \bigcup_{i=1}^{n} A_{i}$ and $A \subseteq \bigcup_{i=1}^{n} A_{i}$. Since $V \subseteq S, s_{0} \in S$. Let $B$ be a closed (in $S$ ) increasing set which is contained in $M_{S}\left(s_{0}\right) \backslash\{s\}$. Since $V$ is convex relative to $T, \bar{B} \cap L_{T}(s)=\varnothing$, where $\bar{B}$ denotes the closure of $B$ in $T$. Since 
$\bar{B}$ is compact, $M_{T}(\bar{B})$ is closed in $T$. Also, $M_{T}(\bar{B}) \subseteq M_{T}\left(s_{0}\right) \backslash\{s\}$. The theorem now follows by the above remarks.

THEOREM 3.6. Let $S$ be a locally compact semilattice which is topologically isomorphic to a subsemilattice of a product of min intervals. Then $S$ is locally convex, $L U$-completely regular, and $L U$-finite.

Proof. By [2], a closed subsemilattice of a product of min intervals has sufficiently many continuous homomorphisms into the min interval to separate points. Thus Theorem 3.6 follows from Lemmas 3.3, 3.4, and 3.5.

Lemma 3.7. Let $S$ be a topological semilattice for which $\operatorname{Hom}(S, M)$ separates points. Then the following are equivalent:

(a) The evaluation map $\Psi$ is an embedding.

(b) For each $s$ in $S$ and each open neighborhood $U$ of $s$ there are a finite number of homomorphisms $f_{1}, \cdots, f_{n}$ in $\operatorname{Hom}(S, M)$ and a finite number of open subsets $O_{1}, \cdots, O_{n}$ of $M$ such that $s \in \bigcap_{i=1}^{n} f_{i}^{-1}\left(O_{i}\right)$ $\subseteq U$.

Proof. (a) $\Rightarrow$ (b) Let $s \in S$ and let $U$ be an open neighborhood of $s$. Since $\Psi$ is an embedding, $\Psi(U)$ is open relative to $\Psi(S)$ and $\Psi(s) \in \Psi(U)$ Thus there is a set $V$ which is open relative to $X_{S} M$ such that $V \cap \Psi(S)=\Psi(U)$. Since $X_{S} M$ has the product topology, there are finite number of homomorphisms $f_{1}, \cdots, f_{n}$ in $\operatorname{Hom}(S, M)$ and a finite number $O_{1}, \ldots, O_{n}$ of open subsets of $M$ such that $\Psi(s) \in\left(\bigcap_{i=1}^{n} \operatorname{pr}_{f_{i}}^{-1}\left(O_{i}\right)\right) \subseteq V$. Thus, it follows that $s \in \bigcap_{i=1}^{n} f_{i}^{-1}\left(O_{i}\right) \subseteq U$.

(b) $\Rightarrow$ (a) Let $U$ be a nonempty open subset of $S$ and let $s \in U$. Let $f_{1}, \cdots, f_{n}$ be elements of $\operatorname{Hom}(S, M)$ and let $O_{1}, \cdots, O_{n}$ be open subsets of $M$ such that $\Psi(s) \in \bigcap_{i=1}^{n} f_{i}^{-1}\left(O_{i}\right) \subseteq U$. It follows that $\Psi(s) \in\left(\bigcap_{i=1}^{n} \operatorname{pr}_{f_{i}}^{-1}\left(O_{i}\right)\right) \cap \Psi(S) \subseteq \Psi(U)$, and that $\Psi(U)$ is open relative to $\Psi(S)$. That $\Psi$ is injective follows since $\operatorname{Hom}(S, M)$ separates points. Thus $\Psi$ is an embedding.

THEOREM 3.8. If $S$ is locally convex, LU-completely regular, and $L U$-finite, then the evaluation map $\Psi$ is an embedding.

Proof. Let $s \in S$ and let $U$ be an open neighborhood of $s$. Since $S$ is locally convex, there is an open convex neighborhood $V$ of $s$ with $V \subseteq U$. Let $s_{0}$ be a point in $V$ satisfying Definition 2.3 with respect to $s$ and let $s_{1} \in M\left(s_{0}\right)^{\circ} \cap V$ satisfying Definition 2.2 with respect to $s$. If $S \backslash M\left(s_{1}\right)^{\circ}=\varnothing$, then let $f_{0}: S \rightarrow M$ be the map defined by $f_{0}(t)=1$ for all $t$ in $S$. If $S \backslash M\left(s_{0}\right)^{\circ} \neq \varnothing$, then by Theorem 3.1 there is an $f_{0}$ in $\operatorname{Hom}(S, M)$ such that $f_{0}(s)=1$ and $f_{0}\left(S \backslash M\left(s_{1}\right)^{\circ}\right)=0$. Let $B$ $=(S \backslash V) \cap M\left(s_{1}\right)$. Since $V$ is convex and $s_{1} \in V, B$ is a closed increas- 
ing set contained in $M\left(s_{1}\right)$ and thus in $M\left(s_{0}\right)$. If $B=\varnothing$, then $f_{0}$ and $(1 / 2,1]$ satisfy the conditions of Lemma 3.7b. If $B \neq \varnothing$, then there are closed increasing subsemilattices $A_{1}, \cdots, A_{n}$ such that $s \notin \bigcup_{i=i}^{n} A_{i}$ and $B \subseteq \bigcup_{i=1}^{n} A_{i}$. Let $B_{i}=A_{i} \cap M\left(s_{1}\right)$. Then each $B_{i} \subseteq M\left(s_{1}\right) \backslash\{s\}$ and $B \subseteq \cup_{i=1}^{n} B_{i}$. Thus there are functions $f_{1}, \cdots, f_{n}$ in $\operatorname{Hom}(S, M)$ such that $f_{i}\left(B_{i}\right)=1$ and $f_{i}(s)=0$. Let $O_{0}=(1 / 2,1]$ and let $O_{i}=[0,1 / 2)$ for $i=1,2, \cdots, n$. Then $s \in \bigcap_{i=0}^{n} f_{i}^{-1}\left(O_{i}\right) \subseteq V \subseteq U$. Thus it follows by Lemma $3.7 \mathrm{~b}$ that $\Psi$ is an embedding.

EXAmple 1. Let $S_{1}=\{(0,1 / n) \mid n$ is a positive integer $\}$ and let $S_{2}=\{(1+1 / n, 0) \mid n$ is a positive integer $\}$. Let $S=S_{1} \cup S_{2} \cup(0,0)$, and define multiplication on $S$ by

$$
\begin{aligned}
\left(0, \frac{1}{n}\right)\left(0, \frac{1}{m}\right) & =\left(0, \min \left\{\frac{1}{n}, \frac{1}{m}\right\}\right), \\
\left(1+\frac{1}{m}, 0\right)\left(0, \frac{1}{n}\right) & =\left(0, \frac{1}{n}\right)\left(1+\frac{1}{m}, 0\right) \\
& =\left(1+\frac{1}{m}, 0\right) \text { if } m \geqq n, \\
& =\left(0, \frac{1}{n}\right) \quad \text { if } n<m, \\
\left(1+\frac{1}{n}, 0\right)\left(1+\frac{1}{m}, 0\right) & =\left(\min \left\{1+\frac{1}{n}, 1+\frac{1}{m}\right\}, 0\right), \\
(0,0) \cdot x & =x \cdot(0,0)=(0,0) \text { for all } x \text { in } S .
\end{aligned}
$$

Then $S$ is a locally compact, $L U$-finite semilattice which is not locally convex or $L U$-completely regular. Thus $S$ is not embeddable in a product of min intervals.

EXAMPLE 2. Let $S=M \times Z$, where $Z$ denotes the nonnegative integers. Define multiplication as follows:

$$
\begin{aligned}
(x, z)(y, w) & =(0,0) & \text { if } z \neq w \\
& =(x y, z) & \text { if } z=w .
\end{aligned}
$$

Then $S$ is a locally compact, locally convex, $L U$-completely regular semilattice that is not $L U$-finite. Note that $S$ is not $L U$-finite at $(0,0)$ since $M \times(Z \backslash\{0\})$ is an upper set contained in $M((0,0))$ which cannot be contained in a finite union upper subsemilattice unless one of the subsemilattices contains $(0,0)$, thus $S$ is not embeddable in a product of min intervals.

We close with the following embedding theorem: 
THEOREM 3.9. Let $S$ be a locally compact semilattice with the property that if $s \in S, U$ is an open neighborhood of $s, x \in U$, and $x<y$, then there is a $z$ in $U$ with $x<z<y$. If $S$ satisfies the hypothesis of Theorem 3.1, then the evaluation map $\Psi$ is an embedding.

Proof. Let $s \in S$, and let $U$ be an open neighborhood of $s$. Let $V$ be an open neighborhood of $s$ with $\bar{V}$ compact and $\bar{V} \subseteq U$. Then $M(V)$ is an open increasing neighborhood of $s$; thus, there is a point $s_{0}$ in $M(V)$ with $s \in M\left(s_{0}\right)^{\circ}$. It follows that there is a point $s_{1}$ in $V$ with $s \in M\left(s_{1}\right)^{\circ}$. We show that there is a point $s_{2}$ in $V$ with $s \in M\left(s_{2}\right)^{\circ}$ such that $M\left(s_{2}\right) \cap L(s) \subseteq V$. Assume that this is not the case, and let $F(s)=\{W \mid W$ is an open neighborhood of $s$ contained in $V\}$. Partially order $F(s)$ by $W_{1} \leqq W_{2}$ if and only if $W_{2} \subseteq W_{1}$; then $F(s)$ with the partial order $\leqq$ is a directed set. For each $W$ in $F(s)$ let $s_{W} \in W$ with $s \in M\left(s_{W}\right)^{\circ}$. Then (by the compactness of $\bar{V}$ ) there is a point $t_{W}$ in $\bar{V} \backslash V$ with $s_{W}<t_{W}<s$. Then the net $\left\{s_{W} \mid W \in F(s)\right\}$ converges to $s$, and the net $\left\{t_{W} \mid W \in F(s)\right\}$ clusters to some point $t_{0}$ in $\bar{V} \backslash V$. It follows that $s \leqq t_{0} \leqq s$ but $s \neq t_{0}$, which is impossible; thus such an $s_{2}$ must exist.

For each $b$ in $M\left(s_{2}\right) \backslash V$ let $s_{b}$ be a maximal element in $M\left(s_{2}\right) \cap \bar{V}$ satisfying $s_{b} \leqq b$ (such elements exist since $\bar{V}$ is compact). Observe that each $s_{b} \in \bar{V} \backslash V$, which is compact. Also, by the above, $s_{b} \notin L(s)$; thus, there is an $f_{b}$ in $\operatorname{Hom}(S, M)$ with $f_{b}\left(s_{b}\right)=1$ and $f_{b}(L(s))=0$ (Theorem 3.1). The set $\left\{f_{b}^{-1}((1 / 2,1]) \mid b \in M\left(s_{2}\right) \backslash V\right\}$ is an open cover of $M\left(s_{2}\right) \cap(\bar{V} \backslash V)$; thus, there is a finite subset $\left\{f_{b_{i}}^{-1}((1 / 2,1)) \mid i=1, \cdots, n\right\}$ which covers $M\left(s_{2}\right) \cap(\bar{V} \backslash V)$. Since $M\left(s_{2}\right)^{\circ}$ is open, $M\left(M\left(s_{2}\right)^{\circ}\right)$ is open. Also, $M\left(M\left(s_{\alpha}\right)^{\circ}\right) \subseteq M\left(s_{\alpha}\right)$. Thus $M\left(s_{2}\right)^{\circ} \subseteq M\left(M\left(s_{2}\right)^{\circ}\right) \subseteq M\left(s_{2}\right)^{\circ}$ which implies $M\left(s_{2}\right)^{\circ}$ is an increasing set; thus $S \backslash M\left(s_{2}\right)^{\circ}$ is a decreasing set. By Theorem 3.1, there is an $f_{0}$ in $\operatorname{Hom}(S, M)$ such that $f_{0}(s)=1$ and $f_{0}\left(S \backslash M\left(s_{2}\right)^{\circ}\right)=0$ (if $S \backslash M\left(s_{2}\right)^{\circ} \neq \varnothing$ ). It follows that $s \in f_{0}^{-1}((1 / 2,1]) \cap\left(\bigcap_{i=1}^{n} f_{b_{i}}^{-1}([0,1 / 2))\right) \subseteq V \subseteq U$; thus, by Lemma 3.7, $\Psi$ is an embedding.

Finally, I wish to thank Professor D. R. Brown for his many helpful suggestions and comments.

\section{REFERENCES}

1. J. L. Kelly, General topology, Van Nostrand, Princeton, N. J., 1955. MR 16, 1136.

2. J. D. Lawson, Topological semilattices with small semilattices, J. London Math. Soc. 1 (1969), 719-724.

3. L. Nachbin, Topology and order, Van Nostrand Math. Studies, no. 4, Van Nostrand, Princeton, N. J., 1965. MR 36 \#2125.

University of Houston, Houston, TeXas 77004 